\title{
URBANIZACIÓN Y
} ENTORNOS ALIMENTARIOS RELACIONADOS A LA SALUD CARDIOVASCULAR EN LA CIUDAD DE LA CALERA, CÓRDOBA,

Vanesa Roxana Scarlatta1, María Daniela Defagó ${ }^{2}$

${ }^{1}$ Lic. en Nutrición. Escuela de Nutrición, Facultad de Ciencias Médicas, Universidad Nacional de Córdoba.,

${ }^{2}$ Dra. en Ciencias de la Salud, Mgter. en Efectividad Clínica, Lic. en Nutrición. Escuela de Nutrición, Universidad Nacional de Córdoba. Facultad de Ciencias Médicas, Instituto de Investigaciones en Ciencias de la Salud (INICSA-CONICET).

DOI: https://doi. org/10.31052/1853.1180. v24n1

@Universidad Nacional de Córdoba

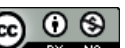

Trabajo recibido: 08 de octubre 2019. Aprobado: 08 de mayo 2020.

\section{ARGENTINA, 2016- 2017.}

URBANIZATION AND EATING SETTINGS RELATED TO CARDIOVASCULAR HEALTH IN THE CITY OF LA CALERA, CORDOBA, ARGENTINA, 2016-2017.

URBANIZAÇÃO E AMBIENTES ALIMENTARES RELACIONADOS À SAÚDE CARDIOVASCULAR NA CIDADE DE LA CALERA, CÓRDOBA, ARGENTINA, 2016-2017

\section{Resumen}

Objetivo: analizar el entorno alimentario a través de la disponibilidad comercial de alimentos asociados a la salud cardiovascular y su relación con el nivel de urbanización en la localidad de La Calera. Metodología: se relevaron las características de urbanización y disponibilidad comercial de alimentos. Se analizó la asociación entre las características de urbanización y la disponibilidad alimentaria según zona. Se georefenció el entorno comercial alimentario con el programa QGIS 2.18.4 Las Palmas. Resultados: las zonas geográficas se categorizaron en: Urbanización nivel 1 (alcanzaron o superaron el $75 \%$ de los indicadores de urbanización estudiados, zonas centro, norte y oeste) y Urbanización nivel 2 (no alcanzaron el $75 \%$ de los indicadores, zonas sur y este). La disponibilidad de margarina $(\mathrm{p}=0,04)$ y pan envasado $(\mathrm{p}=0,01)$ fue mayor en las zonas con menor nivel de ur- 
banización. Conclusiones: los indicadores de urbanización afectaron significativamente la disponibilidad comercial de margarina y panes envasados.

Palabras Clave: Enfermedades cardiovasculares - urbanización - alimentos.

\section{Abstract}

Objective: To analyze eating settings through the commercial availability of products associated with cardiovascular health and their relationship with urbanization level in the city of La Calera. Method: Characteristics of urbanization and food commercial availability were gathered. The association between urbanization characteristics and food availability was analyzed according to areas. The commercial area was geo-referenced with the program QGIS 2.18.4 - Las Palmas. Results: Geographic areas were categorized in: Urbanization Level 1: (reached or exceeded $75 \%$ of urbanization indicators studied; center, west and north areas) and Urbanization Level 2 (did not reach $75 \%$ of indicators, south and east areas). The availability of margarine $(\mathrm{p}=0.04)$ and packaged bread $(\mathrm{p}=0.01)$ was higher in areas with lower level of urbanization. Conclusions: Urbanization indicators significantly affect commercial availability of margarine and packaged bread.

Key Words: cardiovascular diseases - urbanization - food.

\section{Resumo}

Objetivo: analisar o ambiente alimentar por meio da disponibilidade comercial de alimentos associados à saúde cardiovascular e sua relação com o nível de urbanização da cidade de La Calera. Metodologia: foram pesquisadas as características de urbanização e disponibilidade comercial de alimentos. A associação entre características da urbanização e disponibilidade de alimentos por área foi analisada. $\mathrm{O}$ ambiente comercial de alimentos foi georreferenciado com o programa QGIS 2.18.4 - Las Palmas. Resultados: as áreas geográficas foram categorizadas em: Nível de Urbanização 1 (atingiram ou ultrapassaram $75 \%$ dos indicadores de urbanização estudados, áreas central, norte e oeste) e Nível de Urbanização 2 (não atingiram $75 \%$ dos indicadores, áreas sul e leste). A disponibilidade de margarina $(\mathrm{p}=0,04)$ e pão empacotado $(\mathrm{p}=0,01)$ foi maior nas áreas com menor nível de urbanização. Conclusões: os indicadores de urbanização afetaram significativamente a disponibilidade comercial de margarina e pães empacotados.

Palavras chave: doenças cardiovasculares - urbanização - alimentos.

\section{Introducción}

La nutrición adecuada es una necesidad humana básica y una condición indispensable de salud. En este sentido, el desarrollo de estrategias efectivas para el control y promoción de una alimentación y nutrición saludables requiere contar con información precisa y confiable de una amplia gama de factores causales, dentro de los cuales se encuentra el entorno alimentario al cual las personas se encuentran expuestas (1).

El crecimiento de las áreas urbanas se asocia a la adopción de estilos de vida poco saludables que pueden impactar en el riesgo cardiovascular futuro (2). Las influencias globales y los cambios sociales asociados con el crecimiento económico, la modernización y la globalización son factores involucrados las transformaciones en los hábitos de nutrición en todo el mundo, caracterizándose por un aumento en la ingesta de alimentos con alta densidad energética, grasas saturadas, azúcar y sal y de bajo valor nutricional (3).

Argentina se encuentra en una fase de transición demográfica, epidemiológica y nutricional. Los cambios ligados al uso de la sal en los alimentos, una dieta rica en grasas saturadas de origen animal, el consumo de grasas trans y una ingesta excesiva de hidratos 
de carbono refinados aumentan la frecuencia de los principales factores de riesgo que conducen a la aparición de enfermedades crónicas, como las cardiopatías y la diabetes mellitus tipo 2. $(4,5)$. En consonancia, diversas localidades de la provincia de Córdoba han experimentado un crecimiento exponencial en sus poblaciones urbanizadas. En particular, la ciudad de La Calera es una de las que más creció en la última década. Según el Censo del año 2010, esta ciudad presentó un aumento del $29 \%$ en su número de habitantes, duplicando la cantidad de hectáreas de su ejido urbano (6).

La disponibilidad de alimentos saludables y a precios asequibles es reconocida como un factor clave en la elección de alimentos y como parte de las estrategias de prevención de enfermedades en el campo de la salud pública. El concepto de entorno alimentario se refiere a los ambientes, oportunidades y condiciones físicas, económicas, políticas y socioculturales, que enmarca la interacción de los consumidores con el sistema alimentario, generando sugerencias cotidianas sobre la adquisición, preparación y el consumo de alimentos; determinan tanto las preferencias y elecciones alimentarias de las personas como su estado nutricional (7). Estos entornos pueden ser amplios y diversos, con un gran número de opciones alimentarias y escalas de precios, o de manera contraria, con escasez de los mismos y variabilidad. A pesar de que las decisiones individuales son determinantes al momento de adquirir un alimento, el contexto tiene una gran influencia en la configuración de las preferencias alimentarias, por esto, existe una responsabilidad colectiva, de poner en marcha políticas que a través de medidas de diverso tipo (económicas, legislativas, educativas) vayan dirigidas a crear un entorno que facilite las elecciones saludables $(8,9)$. Existe una amplia disponibilidad de alimentos industrializados lo cual representa un riesgo potencial para la salud de los consumidores. El rápido y elevado consumo de los productos ultra-procesados, es la causa alimentaria principal del aumento de la obesidad y otras enfermedades no trasmisibles, como las cardiovasculares. Por otro lado, se encuentran disponibles alimentos cardiosaludables que evitan el desarrollo de estas patologías, como frutas, verduras, legumbres, aceites, pescados, que aportan fibras, vitaminas, minerales y ácidos grasos poliinsaturados (omega-3 y omega-6) y monoinsaturados (omega-9) $(10,11)$.

El mapeo de alimentos o food mapping es un término reciente que se aplica al proceso de investigación acerca de la disponibilidad de alimentos en la población; tiene como objetivo identificar las áreas geográficas o comunidades que tienen las mayores necesidades en términos de acceso a los alimentos, como así también para identificar la disponibilidad de tipos específicos de alimentos (12). Es una oportunidad para el desarrollo de estrategias de promoción de alimentación saludable en base a las necesidades. De este modo, la cartografía de alimentos podría ayudar a lograr un cambio positivo y eficaz frente a las barreras para el acceso a alimentos saludables.

El presente trabajo tuvo como objetivo analizar el entorno alimentario, a nivel de la disponibilidad espacial comercial de alimentos asociados a la salud cardiovascular, y su relación con el área urbanizada en la localidad de La Calera, durante el período 2016 - 2017.

\section{Materiales y Métodos}

Se realizó un estudio de tipo observacional, analítico y de corte transversal. El universo estuvo conformado por todos los comercios expendedores de alimentos de la localidad de La Calera, Córdoba, Argentina, en el periodo 2016- 2017. Para el cálculo del tamaño muestral, se contabilizaron 491 manzanas pertenecientes a zona urbanizada (excluyendo los barrios cerrados y countries).

Para el cálculo de la muestra se utilizó el software G*Power. Se aceptó un riesgo de 0,05 y una potencia estadística del $80 \%$, determinando un tamaño muestral de 81 manzanas. Seguidamente, se seleccionaron aleatoriamente 9 barrios ubicados en diferentes zonas geográficas de la localidad y se aletorizaron 9 manzanas de cada barrio para el relevamien- 
to de los locales expendedores de alimentos.

La primera etapa consistió en muestrear aleatoriamente los puntos de venta en los radios censales de toda la localidad, estratificados por tipología (comercio menor de alimentos: almacenes, kioscos, despensas y dietéticas; comercio mayor de alimentos: autoservicios, supermercados) (13).

Los barrios y zonas geográficas estudiadas fueron definidos según indicadores construidos a partir del análisis de las variables de urbanización adaptadas de la Agencia de Ecología Urbana de Barcelona (14). Para ello, se diseñó una planilla de relevamiento de datos por observación y, en caso de ser necesario, a través de indagación a los vecinos de la zona en cuestión (especialmente en el caso de servicios básicos de uso cotidiano). Las características de urbanización fueron relevadas de las manzanas en la que se encontraban los comercios expendedores de alimentos.

A los propietarios/responsables de los establecimientos seleccionados por el procedimiento de muestreo se les solicitó autorización para ingresar y relevar la disponibilidad de algunos alimentos y se manifestó la ausencia de beneficios por su participación y la protección de los datos personales del comercio de acuerdo a la ley 25.326. La evaluación de la disponibilidad de alimentos se realizó a través de la aplicación de la Encuesta de medición del ambiente nutricional de comercios (NEMS-S, Nutrition Environment Measures Survey for Stores) adaptada, la cual permite la obtención de información acerca de opciones y calidad nutricional de alimentos lácteos, frutas, hortalizas, alimentos enlatados, carnes procesadas, panificados, cereales y misceláneas (15). Se focalizó en los alimentos relacionados a la salud cardiovascular, los cuales incluyeron, en todas sus variedades a los siguientes alimentos: lácteos, carnes, productos de panificación, snacks, frutos secos, aceites, grasas de origen animal, hortalizas, frutas y semillas.

A partir de las características de urbanización relevadas, se categorizó a las zonas geográficas en dos tipos:

- Urbanización nivel 1: alcanzó el 75\% o más de los indicadores de urbanización estudiados.

- $\quad$ Urbanización nivel 2: no alcanzó el 75\% de los indicadores de urbanización estudiados.

Para el análisis de los datos de disponibilidad alimentaria, se construyeron dos categorías de acuerdo a las características nutricionales de los alimentos en relación a la salud cardiovascular: Categoría más saludable: frutas y hortalizas, leche, yogur y quesos descremados, pan envasado/panificados de harina integral, pan fresco, frutas secas, frutas frescas, hortalizas, frutas oleosas, semillas, aceite de oliva, carnes magras, pescados frescos y enlatados; Categoría menos saludable: pan con agregado de grasas (pan criollo, facturas, galletas dulces y saladas o alfajores), snacks (chizitos, palitos, papitas fritas), lácteos enteros, aceites de girasol, maíz y mezclas, manteca, margarina, fiambres, embutidos, otras carnes grasas. En primer lugar, se realizó un análisis descriptivo para la tipificación de las zonas según variables de urbanización y por la disponibilidad de cada uno de los alimentos estudiados. Luego, se aplicó el test exacto de Fisher para analizar la asociación entre la disponibilidad de los alimentos estudiados según tipificación de urbanización y localización geográfica. Además, se desarrolló un modelo de regresión logística para analizar la disponibilidad de alimentos relacionados a la salud cardiovascular según nivel de urbanización, categorizado como presencia/ausencia, ajustado por zona geográfica y tipo de comercio. Se consideró un nivel de significación de $\mathrm{p}<0,05$. El análisis se realizó a través del software Stata v.11.

A partir de la información relevada se realizó, mediante un sistema de GPS (sistema de posicionamiento global, Global Positioning System), la determinación de las coordenadas geográficas de latitud y longitud a fin de confeccionar un mapa para identificar la 
disponibilidad de comercios que expendan alimentos cardiosaludables y culturalmente aceptables. Utilizando como base una imagen satelital actualizada de la ciudad se procedió a geolocalizar, en una capa en formato de puntos, cada uno de los comercios que conformaron la muestra. A esta capa se le adjuntó información respecto del tipo de comercio. Por otro lado, se confeccionó una capa poligonal con datos referidos a las zonas de La Calera y sus niveles de urbanización. Para el mapa de disponibilidad de alimentos cardiosaludables, se utilizó como base un mapa de manzanas y calles de la ciudad disponible a través de un WMS (web map service) y las capas elaboradas anteriormente. Se crearon 2 capas nuevas, de puntos, una de alimentos más saludables y otra de alimentos menos saludables. A esta capa se le asignaron los datos de disponibilidad/no disponibilidad de los mismos para cada uno de los comercios. Para realizar ambos mapas se utilizó el programa QGIS 2.18.4 - Las Palmas.

\section{Resultados}

De la recolección de datos realizada en los distintos barrios de la ciudad de La Calera, se relevaron las características de urbanización que se presentan en la Tabla 1.

Tabla $N^{\circ}$ 1: Características de urbanización según zonas geográficas de la ciudad de La Calera, año 2016 - 2017

\begin{tabular}{|c|c|c|c|c|c|c|}
\hline \multirow{2}{*}{$\begin{array}{l}\text { Características de } \\
\text { urbanización }\end{array}$} & \multicolumn{5}{|c|}{ Zonas } & \multirow{2}{*}{ p-valor } \\
\hline & Centro & Norte & Sur & Este & Oeste & \\
\hline Densidad de viviendas & $\begin{array}{c}+55: 0 \% \\
-55: 100 \%\end{array}$ & $\begin{array}{c}+55: 0 \% \\
-55: 100 \%\end{array}$ & $\begin{array}{c}+55: 0 \% \\
-55: 100 \%\end{array}$ & $\begin{array}{c}+55: 0 \% \\
-55: 100 \%\end{array}$ & $\begin{array}{c}+55: 0 \% \\
-55: 100 \%\end{array}$ & - \\
\hline $\begin{array}{l}\text { Accesibilidad } \\
\text {-Acera c/ pendiente } \\
\text { Si } \\
\text { No } \\
\text {-Acera } 2,5 \mathrm{~m} \text { min. } \\
\text { Si } \\
\text { No }\end{array}$ & $\begin{array}{c}100 \% \\
0 \% \\
100 \% \\
0 \%\end{array}$ & $\begin{array}{c}0 \% \\
100 \% \\
\\
0 \% \\
100 \%\end{array}$ & $\begin{array}{c}0 \% \\
100 \% \\
100 \% \\
0 \%\end{array}$ & $\begin{array}{c}0 \% \\
100 \% \\
0 \% \\
100 \%\end{array}$ & $\begin{array}{c}0 \% \\
100 \% \\
0 \% \\
100 \%\end{array}$ & $\begin{array}{c}0,02 \\
<0,0001\end{array}$ \\
\hline $\begin{array}{l}\text { Espacios verdes } \\
\text {-Presencia arbolado } \\
\text { min. } 75 \% \\
\text { Si } \\
\text { No } \\
\text {-Espacios verdes máx. } \\
300 \mathrm{~m} \\
\text { Si } \\
\text { No }\end{array}$ & $\begin{array}{c}50 \% \\
50 \% \\
100 \% \\
0 \%\end{array}$ & $\begin{array}{c}100 \% \\
0 \% \\
100 \% \\
0 \%\end{array}$ & $\begin{array}{c}0 \% \\
100 \% \\
100 \% \\
0 \%\end{array}$ & $\begin{array}{c}0 \% \\
100 \% \\
0 \% \\
100 \%\end{array}$ & $\begin{array}{c}100 \% \\
0 \% \\
100 \% \\
0 \%\end{array}$ & 0,001 \\
\hline $\begin{array}{l}\text { Movilidad } \\
\text {-Transporte público } \\
\text { máx. 300m } \\
\text { Si } \\
\text { No } \\
\text {-Movilidad ciclista máx. } \\
300 \mathrm{~m} \\
\mathrm{Si} \\
\text { No } \\
\text {-Senda peatonal máx. } \\
300 \mathrm{~m} \\
\mathrm{Si} \\
\text { No }\end{array}$ & $\begin{array}{c}100 \% \\
0 \% \\
\\
100 \% \\
0 \% \\
\\
0 \% \\
100 \%\end{array}$ & $\begin{array}{c}100 \% \\
0 \% \\
\\
\\
0 \% \\
100 \% \\
\\
0 \% \\
100 \%\end{array}$ & $\begin{array}{c}100 \% \\
0 \% \\
\\
0 \% \\
100 \% \\
\\
16 \% \\
84 \%\end{array}$ & $\begin{array}{c}100 \% \\
0 \% \\
\\
0 \% \\
100 \% \\
\\
0 \% \\
100 \%\end{array}$ & $\begin{array}{c}100 \% \\
0 \% \\
\\
0 \% \\
100 \% \\
\\
0 \% \\
100 \%\end{array}$ & 0,02 \\
\hline
\end{tabular}




\begin{tabular}{|c|c|c|c|c|c|c|}
\hline $\begin{array}{l}\text { Actividades comer- } \\
\text { ciales } \\
\text {-Venta de alimentos } \\
\text { máx.300m } \\
\text { Si } \\
\text { No } \\
\text {-Venta de productos far- } \\
\text { macéuticos máx. } 300 \mathrm{~m} \\
\text { Si } \\
\text { No }\end{array}$ & $\begin{array}{c}100 \% \\
0 \% \\
100 \% \\
0 \%\end{array}$ & $\begin{array}{c}100 \% \\
0 \% \\
0 \% \\
100 \%\end{array}$ & $\begin{array}{c}100 \% \\
0 \% \\
100 \% \\
0 \%\end{array}$ & $\begin{array}{c}100 \% \\
0 \% \\
100 \% \\
0 \%\end{array}$ & $\begin{array}{c}100 \% \\
0 \% \\
100 \% \\
0 \%\end{array}$ & $\begin{array}{c}- \\
0,07\end{array}$ \\
\hline $\begin{array}{l}\text { Servicios básicos } \\
\text {-Agua de red } \\
\mathrm{Si} \\
\mathrm{No} \\
\text {-Agua de pozo } \\
\mathrm{Si} \\
\mathrm{No} \\
\text {-Red eléctrica } \\
\mathrm{Si} \\
\mathrm{No} \\
\text {-Gas natural } \\
\mathrm{Si} \\
\mathrm{No} \\
\text {-Gas envasado } \\
\mathrm{Si} \\
\mathrm{No} \\
\text {-Recolección residuos } \\
\mathrm{Si} \\
\mathrm{No}\end{array}$ & $\begin{array}{c}100 \% \\
0 \% \\
0 \% \\
100 \% \\
100 \% \\
0 \% \\
100 \% \\
0 \% \\
0 \% \\
100 \% \\
100 \% \\
0 \%\end{array}$ & $\begin{array}{c}100 \% \\
0 \% \\
0 \% \\
100 \% \\
100 \% \\
0 \% \\
0 \% \\
100 \% \\
100 \% \\
0 \% \\
100 \% \\
0 \%\end{array}$ & $\begin{array}{c}100 \% \\
0 \% \\
0 \% \\
100 \% \\
100 \% \\
0 \% \\
100 \% \\
0 \% \\
0 \% \\
100 \% \\
100 \% \\
0 \%\end{array}$ & $\begin{array}{c}100 \% \\
0 \% \\
0 \% \\
100 \% \\
100 \% \\
0 \% \\
0 \% \\
100 \% \\
100 \% \\
0 \% \\
100 \% \\
0 \%\end{array}$ & $\begin{array}{c}100 \% \\
0 \% \\
0 \% \\
100 \% \\
100 \% \\
0 \% \\
100 \% \\
0 \% \\
0 \% \\
100 \% \\
100 \% \\
0 \%\end{array}$ & $\begin{array}{c}- \\
<0,0001 \\
<0,0001\end{array}$ \\
\hline $\begin{array}{l}\text { Equipamientos pú- } \\
\text { blicos } \\
\text {-Centro de salud máx. } \\
600 \mathrm{~m} \\
\mathrm{Si} \\
\mathrm{No} \\
\text {-Centro educativo máx. } \\
300 \mathrm{~m} \\
\mathrm{Si} \\
\mathrm{No} \\
\text {-Centro cultural/deporti- } \\
\text { vo máx.600m } \\
\mathrm{Si} \\
\mathrm{No}\end{array}$ & $\begin{array}{l}50 \% \\
50 \% \\
\\
50 \% \\
50 \%\end{array}$ & $\begin{array}{c}100 \% \\
0 \% \\
\\
100 \% \\
0 \%\end{array}$ & $\begin{array}{c}100 \% \\
0 \% \\
\\
100 \% \\
0 \%\end{array}$ & $\begin{array}{c}0 \% \\
100 \% \\
\\
100 \% \\
0 \%\end{array}$ & $\begin{array}{c}100 \% \\
0 \% \\
\\
0 \% \\
100 \% \\
\\
\\
50 \% \\
50 \%\end{array}$ & 0,01 \\
\hline
\end{tabular}

Se aplicó el test de Fisher para analizar las variables con diferencias en los porcentajes observados.

$\mathrm{Al}$ analizar la densidad de viviendas, se observó que todas las manzanas relevadas poseían una densidad habitacional inferior a las 55 viviendas/manzana. La variable accesibilidad fue valorada de acuerdo a las características de las aceras. Sólo las zonas centro y sur presentaron acera de 2,5 metros de ancho pavimentada, mientras que el resto de las áreas relevadas no presentaron acera con estas características, encontrándose cercanas a zonas montañosas o rurales. Con respecto a la presencia de pendiente en las aceras, sólo la zona centro presentó acera con pendiente sobre la calle principal. La zona geográfica se asoció a la accesibilidad, en cuanto a la presencia de acera con pendiente $(\mathrm{p}=0,02)$ y acera de 2,5 metros $(\mathrm{p}<0,0001)$.

Se consideraron las dimensiones proximidad a espacios verdes comunes (valor máximo a 300 metros) y presencia deseable de arbolado (mínimo 75\% a lo largo de la acera) para evaluar la variable espacios verdes. Se observó que todas las zonas recorridas presentaron una proximidad máxima de 300 metros a espacios verdes (como costanera o plazas). El $100 \%$ de las zonas norte y oeste presentaron arbolado, mientras que la zona centro presentó un $50 \%$ de arbolado en la proporción deseable en las aceras y las zonas sur y este no alcanzaron el mínimo deseable $(\mathrm{p}=0,001)$. La zona geográfica se asoció a esta última. La movilidad se determinó a través de laproximidad máxima a trasporte público, senda 
peatonal o red ciclista de 300 metros. Todas las áreas relevadas presentaron una cercanía máxima de 300 metros a transporte público, mientras que para la red ciclista sólo se observó en la zona centro. La zona geográfica se asoció de manera estadísticamente significativa a la presencia de red ciclista $(\mathrm{p}=0,02)$. En cuanto a senda peatonal, ésta fue observada sólo en zona sur. No hubo asociación entre senda peatonal y zona geográfica $(p>0,9)$.

Con respecto a la presencia de actividades comerciales, entendiendo como tales a las actividades económicas de uso cotidiano que el ciudadano utiliza casi a diario, se encontró que en todas las zonas relevadas hubo una distancia máxima de 300 metros, en relación específicamente a puntos de venta de alimentos. Con respecto a puntos de venta de productos farmacéuticos, sólo la zona centro presentó una proximidad máxima de 300 metros, mientras que las restantes áreas relevadas presentaron una distancia superior. La zona geográfica no se asoció a la proximidad a puntos de venta de productos farmacéuticos $(\mathrm{p}=0,07)$.

En relación a los servicios básicos, todas las zonas relevadas contaron con agua de red, red eléctrica y recolección de residuos. Sólo la provisión de gas se asoció estadísticamente a la zona geográfica $(\mathrm{p}=0,0001)$. En cuanto al gas natural, las zonas centro, sur y oeste contaron con este servicio, mientras que las zonas norte y este presentaron gas envasado $(\mathrm{p}=0,0001)$. El equipamiento público se valoró por la presencia de centros de salud y centros culturales/deportivos a una proximidad máxima de 600 metros y centros educativos a 300 metros. Se observó que, de los puntos relevados en las zonas norte, sur y oeste, el $100 \%$ se encontraban próximos al centro de salud, en la zona centro sólo $50 \%$ de las manzanas relevadas y en la zona este ninguna se encontraba próxima a un centro de salud $(p=0,05)$.

En cuanto a la proximidad a centros culturales/deportivos, todas las áreas relevadas en las zonas centro, norte y sur presentaron la proximidad deseada, sólo el $50 \%$ de las áreas estudiadas de zona oeste y ninguna de la zona este $(\mathrm{p}=0,05)$. Por otro lado, la zona geográfica estuvo asociada a la proximidad a centros educativos, observándose que todas las áreas de estudio de las zonas norte, sur y este estaban cercanas a los mismos, mientras que sólo el $50 \%$ en la zona centro y ninguno en la región oeste $(\mathrm{p}=0,01)$.

Según las características de urbanización relevadas y detalladas anteriormente, las zonas centro, norte y oeste (más urbanizadas) correspondieron a Urbanización nivel 1, mientras que las zonas sur y este (menos urbanizadas) se categorizaron en Urbanización nivel 2.

Se detectó la presencia de 18 establecimientos ubicados en las distintas zonas estudiadas (17 comercios tipo despensa/almacén y1 supermercado). Sólo los responsables de dos centros no aceptaron participar (uno de zona este y otro de zona norte), por lo que la muestra final quedó constituida por 16 establecimientos. Una vez obtenida la autorización, se procedió a relevar la presencia/ausencia de alimentos relacionados a la salud cardiovascular.

La Tabla 2 presenta los resultados de los alimentos que se encontraron disponibles a la venta según nivel de urbanización.

Tabla $\mathbf{N}^{\circ}$ 2: Disponibilidad comercial de alimentos relacionados a la salud cardiovascular según nivel de urbanización, ciudad de La Calera, Córdoba, año 2016 - 2017

\begin{tabular}{|l|c|c|c|}
\hline $\begin{array}{c}\text { Alimentos relacionados a } \\
\text { la salud cardiovascular }\end{array}$ & $\begin{array}{c}\text { Nivel de urbanización 1 } \\
\text { (zonas centro, norte y oeste) }\end{array}$ & $\begin{array}{c}\text { Nivel de urbanización 2 } \\
\text { (zonas sur y este) }\end{array}$ & p-valor \\
\hline Lácteos enteros & $\begin{array}{c}100 \% \\
\mathrm{Si}\end{array}$ & $\begin{array}{c}100 \% \\
0 \%\end{array}$ & - \\
No & $67 \%$ & $100 \%$ & \\
\hline Lácteos descremados & $33 \%$ & $0 \%$ & 0,21 \\
$\mathrm{Si}$ & No & & \\
\hline
\end{tabular}




\begin{tabular}{|c|c|c|c|}
\hline $\begin{array}{l}\text { Carne de vaca, pollo } \\
\text { do magra } \\
\mathrm{Si} \\
\text { No }\end{array}$ & $\begin{array}{l}56 \% \\
44 \%\end{array}$ & $\begin{array}{l}86 \% \\
14 \%\end{array}$ & 0,31 \\
\hline $\begin{array}{l}\text { Carne de vaca, pollo } \\
\text { do grasa } \\
\text { Si } \\
\text { No }\end{array}$ & $\begin{array}{c}0 \% \\
100 \%\end{array}$ & $\begin{array}{l}14 \% \\
86 \%\end{array}$ & 0,44 \\
\hline $\begin{array}{l}\text { Pescado } \\
\text { Si } \\
\text { No }\end{array}$ & $\begin{array}{l}89 \% \\
11 \%\end{array}$ & $\begin{array}{c}100 \% \\
0 \%\end{array}$ & 0,99 \\
\hline $\begin{array}{l}\text { Frutas secas } \\
\mathrm{Si} \\
\text { No }\end{array}$ & $\begin{array}{l}67 \% \\
33 \%\end{array}$ & $\begin{array}{l}57 \% \\
43 \%\end{array}$ & 0,99 \\
\hline $\begin{array}{l}\text { Frutas oleosas } \\
\mathrm{Si} \\
\text { No }\end{array}$ & $\begin{array}{l}89 \% \\
11 \%\end{array}$ & $\begin{array}{c}100 \% \\
0 \%\end{array}$ & 0,99 \\
\hline $\begin{array}{l}\text { Aceite de oliva } \\
\text { Si } \\
\text { No }\end{array}$ & $\begin{array}{l}33 \% \\
67 \%\end{array}$ & $\begin{array}{l}71 \% \\
29 \%\end{array}$ & 0,32 \\
\hline $\begin{array}{l}\text { Otros aceites } \\
\mathrm{Si} \\
\text { No }\end{array}$ & $\begin{array}{l}89 \% \\
11 \%\end{array}$ & $\begin{array}{c}100 \% \\
0 \%\end{array}$ & 0,99 \\
\hline $\begin{array}{l}\text { Manteca } \\
\text { Si } \\
\text { No }\end{array}$ & $\begin{array}{c}100 \% \\
0 \%\end{array}$ & $\begin{array}{l}86 \% \\
14 \%\end{array}$ & 0,44 \\
\hline $\begin{array}{l}\text { Margarina } \\
\text { Si } \\
\text { No }\end{array}$ & $\begin{array}{l}22 \% \\
78 \%\end{array}$ & $\begin{array}{l}86 \% \\
14 \%\end{array}$ & 0,04 \\
\hline $\begin{array}{l}\text { Margarinasin trans } \\
\text { Si } \\
\text { No }\end{array}$ & $\begin{array}{l}22 \% \\
78 \%\end{array}$ & $\begin{array}{l}43 \% \\
57 \%\end{array}$ & 0,60 \\
\hline $\begin{array}{l}\text { Panificados con agre } \\
\text { de grasas } \\
\mathrm{Si} \\
\text { No }\end{array}$ & $\begin{array}{c}100 \% \\
0 \%\end{array}$ & $\begin{array}{c}100 \% \\
0 \%\end{array}$ & - \\
\hline $\begin{array}{l}\text { Snacks } \\
\text { Si } \\
\text { No }\end{array}$ & $\begin{array}{c}100 \% \\
0 \%\end{array}$ & $\begin{array}{c}100 \% \\
0 \%\end{array}$ & - \\
\hline $\begin{array}{l}\text { Frutas frescas } \\
\mathrm{Si} \\
\text { No }\end{array}$ & $\begin{array}{l}33 \% \\
67 \%\end{array}$ & $\begin{array}{l}29 \% \\
71 \%\end{array}$ & 0,99 \\
\hline $\begin{array}{l}\text { Frutas envasadas } \\
\mathrm{Si} \\
\text { No }\end{array}$ & $\begin{array}{c}100 \% \\
0 \%\end{array}$ & $\begin{array}{l}20 \% \\
80 \%\end{array}$ & 0,15 \\
\hline $\begin{array}{l}\text { Hortalizas frescas } \\
\mathrm{Si} \\
\text { No }\end{array}$ & $\begin{array}{l}50 \% \\
50 \%\end{array}$ & $\begin{array}{l}40 \% \\
60 \%\end{array}$ & 0,59 \\
\hline $\begin{array}{l}\text { Hortalizas envasadas } \\
\mathrm{Si} \\
\text { No }\end{array}$ & $\begin{array}{l}50 \% \\
50 \%\end{array}$ & $\begin{array}{l}60 \% \\
40 \%\end{array}$ & 0,28 \\
\hline $\begin{array}{l}\text { Semillas } \\
\text { Si } \\
\text { No }\end{array}$ & $\begin{array}{c}0 \% \\
100 \%\end{array}$ & $\begin{array}{c}0 \% \\
100 \%\end{array}$ & - \\
\hline
\end{tabular}




\begin{tabular}{|l|c|c|c|}
\hline Pan fresco & $100 \%$ & $100 \%$ & \\
Si & $0 \%$ & $0 \%$ & \\
No & & $100 \%$ & 0,01 \\
\hline Pan envasado & $40 \%$ & $0 \%$ & \\
Si & $60 \%$ & & \\
No & & & \\
\hline
\end{tabular}

Se aplicó el test de Fisher para analizar las variables con diferencias en los porcentajes observados.

Sólo se encontraron asociaciones estadísticamente significativas en la disponibilidad de los alimentos relacionados a la salud cardiovascular estudiados según nivel de urbanización para margarina $(\mathrm{p}=0,04)$ y pan envasado $(\mathrm{p}=0,01)$, ambos con mayor disponibilidad en la zona menos urbanizada.

Posteriormente, se desarrolló un modelo de regresión logística para analizar la disponibilidad de alimentos relacionados a la salud cardiovascular según nivel de urbanización, ajustado por zona geográfica y tipo de comercio, sin encontrarse asociaciones estadísticamente significativas.

Tabla No 3: Regresión logística para disponibilidad de alimentos asociados a la salud cardiovascular según nivel de urbanización

\begin{tabular}{|c|c|c|c|}
\hline $\begin{array}{c}\text { Nivel urbanización } \\
\text { Alimentos }\end{array}$ & OR & p-valor & IC 95\% \\
\hline Carne magra & 0,29 & 0,36 & $0,02-4,07$ \\
Pescado & 1,35 & 0,74 & $0,21-8,53$ \\
Frutas frescas & 3,12 & 0,39 & $0,22-4,39$ \\
Frutas secas & 0,94 & 0,96 & $0,09-9,28$ \\
Frutas oleosas & 1,35 & 0,74 & $0,21-8,53$ \\
Hortalizas totales & 4,21 & 0,16 & $0,34-5,11$ \\
Aceite de oliva & 0,03 & 0,14 & $0,004-3,05$ \\
Otros aceites & 1,35 & 0,74 & $0,21-8,53$ \\
\hline
\end{tabular}

*Los alimentos que no se presentan es debido a falta de observaciones suficientes.

La Figura 1: presenta el mapa final sobre distribución espacial de alimentos relacionados a la salud cardiovascular, el cual permite identificar geográficamente el entorno alimentario en relación a los alimentos disponibles y no disponibles comercialmente.

En la zona menos urbanizada se observó mayor disponibilidad de los alimentos menos saludables (79\%) en relación a la disponibilidad de alimentos más saludables (61\%). Lo mismo pudo observarse en la zona más urbanizada, aunque con una diferencia menos marcada, encontrándose disponibles un $67 \%$ de alimentos menos saludables y un $57 \%$ de los alimentos más saludables. También puede observarse que en la zona menos urbanizada se encontró una mayor disponibilidad de alimentos en general que en la zona más urbanizada. 


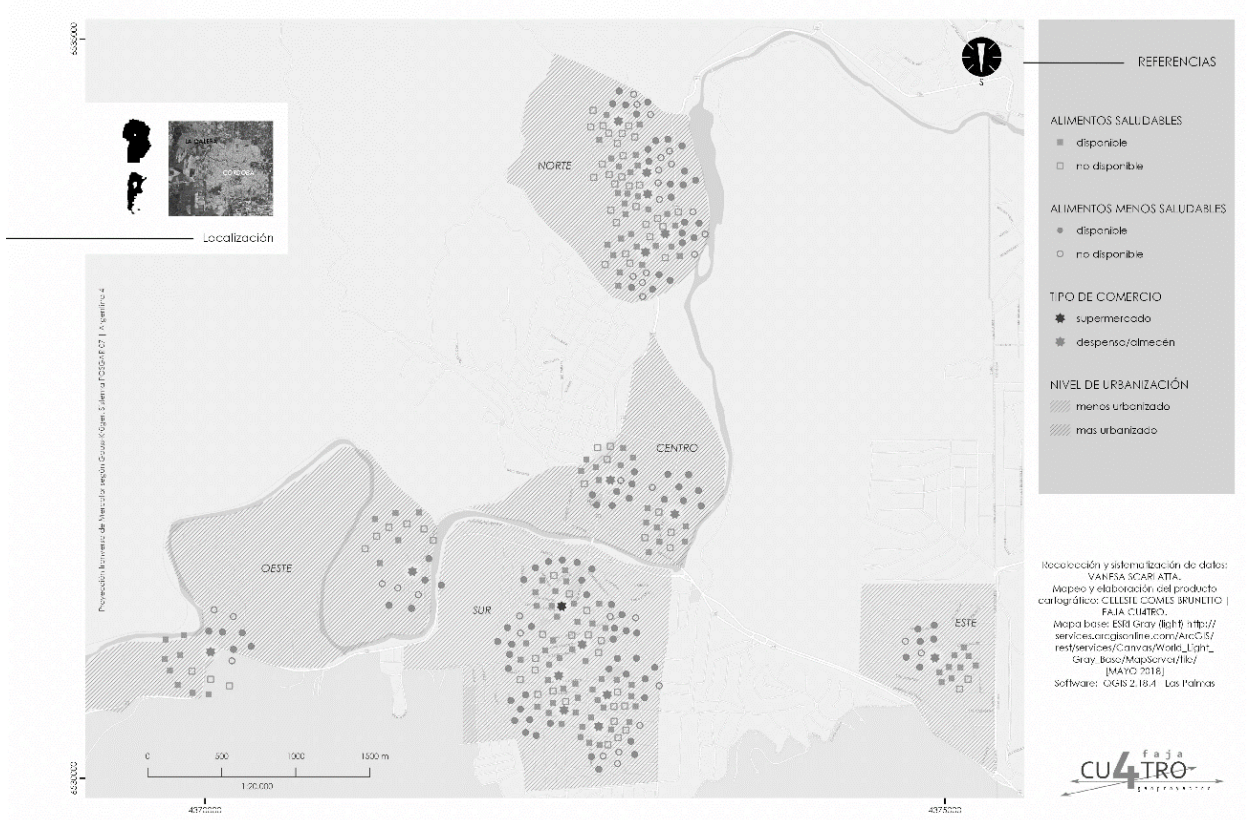

Figura $\mathbf{N}^{\circ}$ 1: Distribución espacial de alimentos relacionados a la salud cardiovascular, ciudad de La Calera, 2016 - 2017

\section{Discusión}

El objetivo del presente trabajo fue realizar un estudio exploratorio para proporcionar una caracterización, según indicadores de urbanización, de distintos sectores de la localidad de La Calera (Córdoba, Argentina), en relación con la salud cardiovascular. Se analizó la disponibilidad comercial de alimentos asociados a la salud cardiovascular y su relación, en el período 2016-2017, con el nivel de urbanización en dicha localidad.

La superficie urbana de Córdoba se expande claramente hacia el oeste y noroeste. El departamento Colon, según censo 2010, estuvo entre los que más crecieron y los más poblados. El crecimiento urbano que se observa en la localidad de estudio refleja un nuevo medio, edificado sobre ecosistemas naturales y generando impactos variados para el medio ambiente y la población de La Calera. Esta expansión es observada también en una gran cantidad de ciudades de América Latina (16). La ciudad tradicional se transforma en un espacio cada vez más discontinuo, disperso y fragmentado, donde los procesos de dispersión de la población están creando nuevos espacios residenciales de bajas densidades y una heterogénea densidad de viviendas (17).

Luego de analizar la disponibilidad de alimentos en los distintos comercios de las zonas estudiadas se observó, en relación a los lácteos y derivados, que estos estuvieron disponibles en todos los comercios de las zonas en estudio. Argentina se caracteriza por ser un país productor de alimentos de origen animal, como lácteos y carnes, que forman parte de su patrón de consumo (16). Con respecto a los lácteos, las empresas ofrecen a los mercados una amplia variedad, con diferentes proporciones de materia grasa, fortificación con ciertos componentes y/o agregado de sustancias modificadoras de sabor y texturas; constituyendo el cuarto grupo de alimentos con mayor participación en el mercado (18).

Con respecto a las carnes, se encontró disponible una mayor cantidad de carne de vaca en relación a la de pollo, y la carne de cerdo sólo se encontró en el supermercado. Sin embargo, en un trabajo realizado por el Centro de Estudios Sobre Nutrición Infantil (CESNI) de Argentina, se mostró una disminución en la disponibilidad de carne vacuna, aproximada- 
mente 20 kilos menos por año por persona, compensada por el aumento de las carnes de pollo y pescado (19). En relación al pescado (fresco y enlatado), estuvo presente en todas las zonas estudiadas, principalmente en sus formas comerciales enlatadas. Sin embargo, a pesar de su disponibilidad, el suministro de energía alimentaria argentino está constituido sólo por $1 \%$ de pescados y frutos de mar (20).

El aceite de oliva es rico en ácido graso oleico (monoinsaturado), y se han comprobado sus efectos beneficiosos cardiovasculares por aumento en los niveles de colesterol HDL, prevención de aterosclerosis y por sus propiedades antioxidantes (21). Éste se encontró en el $80 \%$ de las zonas relevadas, mientras que los otros tipos de aceites se encontraron en todas las zonas. Un estudio realizado por el CESNI, mostró que la disponibilidad de aceites presentó un aumento del 66\%, comparando los volúmenes con los disponibles en los años ' 60 y '70 (19). Por otro lado, los frutos secos que se encontraron fueron nuez, castaña y maní, en un promedio del $60 \%$ en cada una de las zonas geográficas. Los frutos secos se caracterizan por ser fuente de ácidos grasos monoinsaturados y poliinsaturados omega 3 y 6 , lo que mejora el perfil lipídico y disminuye el riesgo de padecer enfermedades cardiovasculares (ECV) (22). Actualmente el mercado nacional de los frutos secos está en constante expansión, no sólo ha incrementado el consumo, sino que lo ha diversificado y desestacionalizado.

En relación a los productos de panificación, estuvieron disponibles en todas las zonas, en forma de criollos, facturas, galletas y alfajores. Estos alimentos contienen altas concentraciones de ácidos grasos saturados y grasas trans, que impactan negativamente en la salud cardiovascular. También son ricos en colesterol, azúcares simples y sodio, por lo que han sido asociados a un aumento en las concentraciones de moléculas inflamatorias y células de adhesión endotelial (23). Estudios realizados en Argentina sobre la disponibilidad de alimentos en la población, muestran un incremento sostenido en la disponibilidad y en la demanda en los rubros galletitas, alfajores y golosinas (24).

Los snacks se encontraron presentes en todas las zonas relevadas en este estudio, sin diferencias según zona. Estos productos se asocian a un aumento en el riesgo de padecer ECV, debido a que son alimentos con alto grado de industrialización y contienen normalmente un alto porcentaje de grasa saturada, trans, sodio y aditivos alimentarios. En nuestro país la industria alimentaria ofrece cada vez más variedad de alimentos, ricos en sabor y también en energía, grasas y azúcares, destacándose los snacks, que se venden normalmente a un costo accesible (25).

Al analizar la disponibilidad de frutas, hortalizas y semillas según zona, alimentos cardioprotectores principalmente por su contenido en fibra, vitaminas, minerales y compuestos fotoquímicos, no se encontró asociación entre la disponibilidad de estos alimentos y zona geográfica, aunque su presencia no fue plena. Por otro lado, al analizar la disponibilidad de alimentos según nivel de urbanización se observó asociación para los alimentos margarina y pan envasado, con mayor presencia en las zonas menos urbanizadas. Dentro de los alimentos manufacturados, los panes industriales presentan un bajo porcentaje de grasa total y porcentajes variados de ácidos grasos trans y poli insaturados según su tipo (24). Con respecto a las margarinas, productos industriales obtenidos a partir de la hidrogenación de aceites vegetales y marinos, son fuertemente cuestionadas por los isómeros trans formados como producto de los procesos de hidrogenación con potencial aterogénico (26). Una de las limitaciones de este trabajo es que, debido a la escasez de estudios locales que midan las características específicas de los entornos urbanos, para este trabajo se utilizó una herramienta de España, tratando de hacer la menor cantidad de adaptaciones posibles para de esta manera mejorar la comparabilidad con otros estudios similares. Sin embargo, una de las fortalezas es que éste es el primer estudio que analiza de manera dinámica la disponibilidad comercial de alimentos relacionados a la salud cardiovascular y el entorno urbano, visibilizándolo a través de mapas o mapeo alimentario. 
En este trabajo, se encontró un entorno alimentario muy variado; y se observó que el proceso de urbanización acontecido en la ciudad de La Calera se asoció a la disponibilidad comercial de margarina y panes envasados. Si bien no se encontraron otras asociaciones estadísticamente significativas, es importante destacar la amplia disponibilidad de alimentos que repercuten negativamente en la salud cardiovascular por su elevado contenido en grasas saturadas y trans, como panificados con agregado de grasa y snacks en todos los puntos muestrales, como se muestra a través del georeferenciamiento.

Finalmente, se destaca la integración de herramientas provenientes de la epidemiología y geomática, brindando un nuevo abordaje para el estudio del entorno alimentario en la enfermedad cardiovascular.

\section{Bibliografia}

1. Trowbridge FL, Wong FL, Byers TE, Serdula MK. Methodological issues in nutrition surveillance: the CDC experience. J Nutr. 1990; 120 Suppl 11:1512-8.

2. Uauy R, Albala C, Kain J. Obesity Trends in Latin America: Transiting from UnderTo Overweight. Journal Nutr 2001; 131:893S-9S.

3. NCD Alliance, 2011. Non-communicable diseases: A Priority for Women's Health and development. [pdf] Geneva: The NCD Alliance. [Consultado: noviembre de 2018]. Disponible en: http://www.who.int/pmnch/topics/maternal/2011_women_ ncd_report.pdf.pdf.

4. Balaguer VI. Control y prevención de las enfermedades cardiovasculares en el mundo. Rev Esp Cardiol. 2004; 57:487-94.

5. Giugliano D, Esposito K. Mediterranean diet and metabolic diseases. Current Opinion in Lipidology. 2008; 19:63-8 10.

6. Colautti, F. La Calera, la que más creció en 10 años. La Voz del Interior. Córdoba, Argentina: 2013. [Consultado: noviembre de 2014]. Disponible en: http://www.lavoz.com.ar/cordoba/calera-que-mas-crecio-10-anos.

7. Caron P, Kalafatic C, Allahoury A. La nutrición y los sistemas alimentarios. Roma: FAO; 2018. Disponible en: http://www.fao.org/3/I7846ES/i7846es.pdf

8. Food and Agriculture Organization (FAO). Influir en los entornos alimentarios en pro de dietas saludables. 2016. Disponible en: http://www.fao.org/3/a-i6491s.pdf

9. Coral CBS, Carmen GC, Consuelo LN, Bricia LP. Manual de alimentación. Planificación alimentaria. Madrid: Editorial UNED; 2016.

10. Zehnder BC. Sodio, potasio e hipertensión arterial. Rev. Med. Clin. CONDES. 2010; 21(4): 510.

11. Organización Mundial de la Salud/Organización Panamericana de la Salud. Alimentos y bebidas ultraprocesados en América Latina: Tendencias, efecto sobre la obesidad en implicaciones para las políticas públicas. 2015. Disponible en: https:// iris.paho.org/bitstream/handle/10665.2/7698/9789275318645_esp.pdf?seque

12. Ball K y Thornton L. Food environments: measuring, mapping, monitoring and modifying. Public Health Nutr. 2013; 16:1147-50.

13. Agencia Santafesina de Seguridad Alimentaria. 2014. https://www.assal.gov.ar/ assa/documentacion/orden_012_14-tasa-localregional.pdf

14. Ministerio de Medio Ambiente y Medio Rural y Marino. Gobierno de España. Sistema de indicadores y condicionantes para ciudades grandes y medianas [Internet]. 2016. [Consultado: julio 2019]. Disponible en: http://www.upv.es/contenidos/CAMUNISO/info/U0722854.pdf

15. Glanz K, Sallis JF, Saelens BE, Frank LD. Nutrition Environment Measures Survey in stores (NEMS-S): development and evaluation. Am J Prev Med. 2007; 32:282-9

16. Food and Agriculture Organization (FAO). Depósito de documentos de la FAO. La 
globalización, la urbanización y la evolución de los sistemas alimentarios en los países en desarrollo. 2016. [Consultado: junio 2019]. Disponible en: http://www. fao.org/docrep/007/y5650s/y5650s04.htm

17. Puebla JG. La ciudad dispersa: cambios recientes en los espacios residenciales de la Comunidad de Madrid. AGUC. 2007; 27:45-67.

18. Lacaze V. Las regulaciones de los alimentos y consumidores: estudio de caso en el sector lácteo de la Argentina actual [Tesis]. Facultad Latinoamericana de Ciencias Sociales; 2008.

19. Centro de Estudio Sobre Nutrición Infantil. Obesidad en Argentina: ¿Hacia un nuevo fenotipo? 2004. [Consultado: noviembre 2018]. Disponible en: http://www.bvsde.ops-oms.org/texcom/cd045364/obesarg.pdf

20. Couseiro ME. La alimentación como un tiempo de la nutrición, su disponibilidad y accesibilidad económica. Rev Cubana Salud Pública. 2007. 33:1-6.

21. Nettleton JA, Steffen LM, Mayer-Davis EJ, Jenny NS, Jiang R, Herrington DM, et al. Dietary patterns are associated with biochemical markers of inflammation and endothelial activation in the Multi-Ethnic Study of Atherosclerosis (MESA). Am J Clin Nutr.2006. 83:1369-1379.

22. Ministerio de Agricultura y Pesca, Alimentación y Medio Ambiente [Internet]. Nuez. 2016. [Consultado: noviembre 2018]. Disponible en: http://www.mapama. gob.es/es/ministerio/servicios/informacion/nuez_tcm7-315322.pdf

23. Mozaffarian D, Clarke R. Quantitative effects on cardiovascular risk factors and coronary heart disease risk of replacing partially hydrogenated vegetable oils with other fats and oils. Eur J Clin Nutr.2009. 63:S22-S33.

24. Ministerio de Agroindustria. Informe de Producto: Galletitas y Bizcochos. 2016. [Consultado: noviembre 2018]. Disponible en: http://www.alimentosargentinos. gob.ar/contenido/sectores/farinaceos/Productos/GalletitasBizcochos_2011_12Dic. pdf

25. Peterson G, Aguilar D, Espeche M, Mesa M, Jáuregui P, Díaz H. Ácidos grasos trans en alimentos consumidos habitualmente por los jóvenes en Argentina. Rev Chil Pediatr. 2006. 77:92-101.

26. Valenzuela BA, Yáñez, CG y Golusda VC. ¿Mantequilla o margarina?: diez años despues. Revista chilena de nutrición, 2010;37:505-513. 